\title{
The Research and Development Program towards an Energy-Frontier Muon Collider
}

\author{
Gail G. Hanson ${ }^{* \dagger}$ \\ Department of Physics and Astronomy, University of California, Riverside \\ Riverside, California 92521 U.S.A. \\ E-mail: Gail.Hansonecern.ch
}

\begin{abstract}
The physics potential of a high-energy lepton collider has been shown to be extraordinary. This facility is capable of detailed studies of potential new physics uncovered at the LHC and can extend the search to mass scales beyond those accessible at the LHC. The Muon Collider provides a possible realization of a multi-TeV lepton collider. A muon accelerator facility that leads to a multi- $\mathrm{TeV}$ Muon Collider presents the unique opportunity to explore new physics within a number of distinct programs that can be brought online as the facility evolves. This paper will give an introduction to the Muon Collider facility and its unique capabilities, will discuss the Research and Development program that strives to make it a reality and finally will briefly give some detail regarding the machine-detector interface, background issues and detector performance.
\end{abstract}

35th International Conference of High Energy Physics (ICHEP2010)

Paris,France

July 22-28, 2010

\footnotetext{
Speaker

$\dagger$ On behalf of the Neutrino Factory and Muon Collider Collaboration (NFMCC), Muon Collider Task Force (MCTF), and Muon Accelerator Program (MAP).
} 


\section{Introduction and History}

Colliding $\mu^{+}$and $\mu^{-}$beams in a storage ring have the same physics potential as an $e^{+} e^{-}$ collider. Muon Colliders were first proposed by G. I. Budker and A. N. Skrinsky in the late 1960's and early 1970's. The necessary concept of ionization cooling was developed by Skrinsky and V. V. Parkhomchuk and expanded by D. Neuffer in the early 1980's and later by R. Palmer [1]. The Muon Collider Collaboration was formed in 1996, and with the addition of the Neutrino Factory [2] became the Neutrino Factory and Muon Collider Collaboration (NFMCC) in 1999. The Fermilab Muon Collider Task Force (MCTF) was formed in 2006. U.S. NFMCC and MCTF activities are now being merged into a new National Muon Accelerator Program (MAP), hosted at Fermilab [1].

\section{Physics Motivation}

Muons are fundamental particles, so they have the same advantage in colliders as electrons: the full center-of-mass energy of the colliding particles is available. Muons have an advantage over electrons since synchrotron radiation is reduced by a factor of $\left(m_{\mathrm{e}} / m_{\mu}\right)^{4} \approx 6 \times$ $10^{-10}$. Therefore a multi-TeV Muon Collider can be compact, with multi-pass acceleration and lower cost for RF power. A high-energy Muon Collider can be much smaller than an electronpositron collider of the same energy, and storage rings can still be used even for multi-TeV colliders. The high-energy muons can travel around a ring 1000 times before decaying, producing collisions with each pass. The muon beam can also have a narrow energy spread.
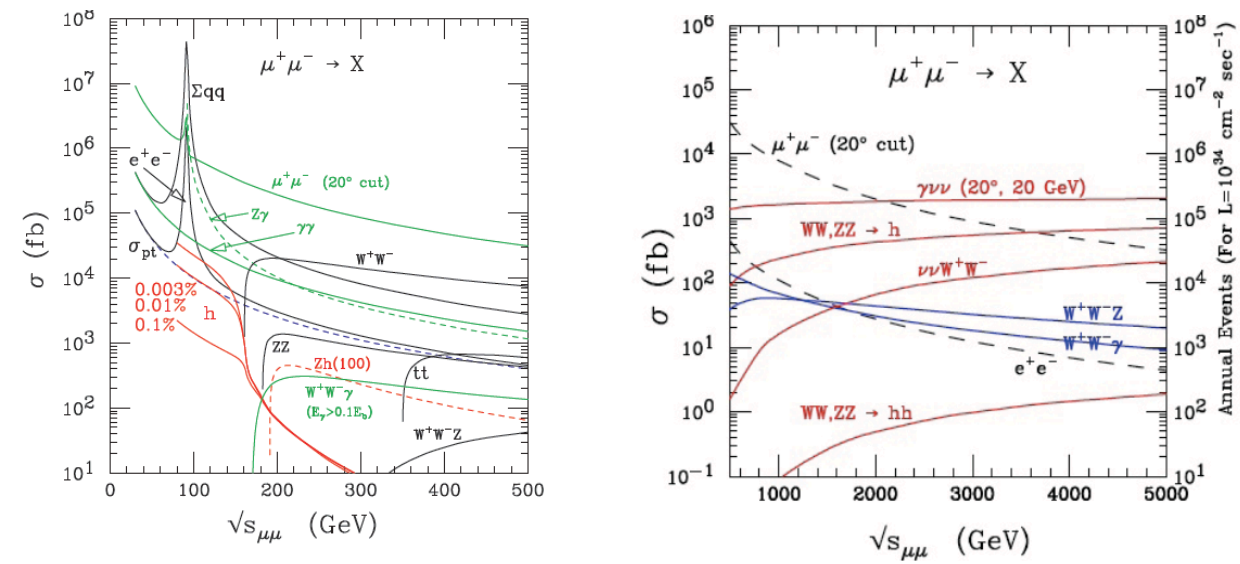

Figure 1: Standard model cross sections for $\mu^{+} \mu^{-}$collisions.

Standard model cross sections for $\mu^{+} \mu^{-}$collisions are shown in Fig. 1. The threshold regions for top-quark pairs, electroweak boson pairs, and $Z h$ production lie in the center-of-mass energy range less than about $500 \mathrm{GeV}$. There is enhanced $s$-channel production for Higgs-like particles, proportional to $\left(m_{\mu} / m_{\varepsilon}\right)^{2} \approx 4 \times 10^{4}$. The narrow energy spread can be used to resolve nearly degenerate states, which could be important for $H^{0}$ and $A^{0}$. 
For center-of-mass energies greater than about $500 \mathrm{GeV}$, fusion processes increasingly dominate $s$-channel processes, allowing all major outstanding questions to be probed.

\section{Progress and Future R\&D}

The same front-end design can be used for the Neutrino Factory and the Muon Collider in the current baseline design, shown in Fig. 2. Since the Muon Collider is compact, a multi-TeV Muon Collider fits on the existing Fermilab site.

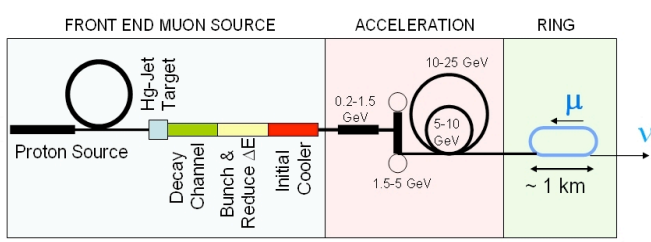

\section{NEUTRINO FACTORY}

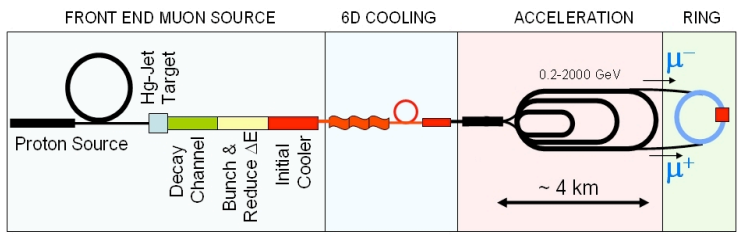

\section{MUON}

COLLIDER

Figure 2: Baseline layouts for Neutrino Factory and Muon Collider.

The proton source is an upgraded Project-X design [3], with a 4-MW beam and 1-3 ns bunch length. The target is a liquid-mercury jet in a 15-T solenoid; a prototype has been operated in the MERIT Experiment at the CERN PS. The muons are produced from the decay of pions produced in the target, so the muon beam has large emittances and energy spreads. The front end captures the pions, bunches the muons, and reduces the energy spread. The decay and capture channel uses the Neutrino Factory Feasibility Study 2 solenoid channel. The Neuffer 12-bunch scheme for bunching and phase rotation is suitable for either a Neutrino Factory or a Muon Collider. The initial cooling follows the design of the Neutrino Factory Feasibility Study 2a channel with lithium hydride instead of liquid hydrogen. For the front end, further R\&D in common with that for the International Design Study for the Neutrino Factory (IDS-NF) is necessary to make this design realistic. In particular, $R \& D$ on $R F$ in high magnetic fields is needed. Normal-conducting copper RF cavities have been shown to break down in multi-Tesla fields at lower gradients than needed for the proposed cooling channels.

There are three options being considered for the six-dimensional cooling: the "Guggenheim" (helical RFOFO), the FOFO snake, and the Helical Cooling Channel. Each has been simulated, and the choice is expected to be made in 2012, with a demonstration proposal in 2016. Again, R\&D on RF in high magnetic fields is needed.

For the final cooling, a channel employing 50-T solenoids and liquid hydrogen absorbers is proposed; R\&D on very high field magnets is needed. The low-energy acceleration consists of a linac followed by two dog-bone RLAs and an FFAG, in common with the IDS-NF baseline design. Acceleration to high energy is accomplished in fast-cycling synchrotrons; R\&D on rapid-cycling magnets is ongoing. The lattice design for the collider ring has achieved $\pm 1.2 \%$ momentum acceptance and $4.7 \sigma$ dynamice aperture (without errors). The collider ring design is closely tied to the design of the detectors. 


\section{R\&D Program}

The U.S. Department of Energy encouraged Fermilab to host a national R\&D program to address the technical challenges and feasibility issues relevant to the capabilities needed for future Neutrino Factory and multi-TeV Muon Collider facilities. A Muon Accelerator Program (MAP) proposal covering R\&D over a 6-7 year period and including 214 participants and 14 institutes was submitted March 1, 2010, and a review took place at Fermilab August 24-26, 2010. MAP deliverables are a Design Feasibility Study Report (DFSR) for a multi-TeV Muon Collider, including an indicative cost range; technology development and system tests needed to inform the Muon Collider DFSR studies and enable down-selection; and contributions to the IDS-NF to produce a Reference Design Report by 2013. Configurations and end-to-end simulation for the Muon Collider are projected for 2014.

\section{Physics and Detector Studies}

Physics and Detector studies are not a part of MAP. A separate group is forming, and a kick-off workshop was held at Fermilab in November 2009. The Machine-Detector Interface group revisited previous background calculations using a consistent Muon Collider lattice and different cone configurations. Compared with the most optimistic old 1996 configuration, peak values for backgrounds are reduced by a factor of 5 to 10 for all particles except photons. Background fluxes of particles are provided as input to physics simulations. As an example, the total absorbed radiation dose per year in silicon at a radius of $4 \mathrm{~cm}$ is comparable to that of CMS at the LHC at a luminosity of $10^{34} \mathrm{~cm}^{-2} \mathrm{~s}^{-1}$.

\section{Summary and Conclusions}

There has been considerable progress on Muon Collider R\&D. A Muon Accelerator Program hosted by Fermilab is being formed, with a Design Feasibility Study and cost range for a multi-TeV Muon Collider within 6-7 years. A plan has been initiated to form a national lepton collider program for physics and detectors in the U.S. The decision on the energy for the next lepton collider will be made $\sim 2014$ based on discoveries at the LHC.

\section{Acknowledgements}

The author wishes to acknowledge the collaboration with many colleagues in NFMCC, MCTF, and MAP, and support from the U.S. Department of Energy grant number DE-FG02$07 E R 41487$.

\section{References}

[1] R\&D Proposal for the National Muon Accelerator Program, FERMILAB-TM-2459-APC, and references therein, https://mctf.fnal.gov/mapproposal.pdf/view

[2] K. Long, these proceedings.

[3] R. Tschirhart, these proceedings. 